\title{
The role and mechanisms of action of SIRT6 in the suppression of postoperative epidural scar formation
}

\author{
XIAOCHEN FAN ${ }^{1 *}$, JUNJIE CHEN $^{2 *}$, DA SHI $^{1}$, JING JIA $^{1}$, \\ JIA HE $^{1}$, LIANG LI ${ }^{1}$, TAO LEI ${ }^{1}$ and XUESONG CHEN ${ }^{1}$
}

\begin{abstract}
${ }^{1}$ Department of Traditional Chinese Medicine Orthopedics Diagnosis and Treatment Center, Honghui Hospital, Xi'an Jiaotong University Health Science Center, Xi'an, Shaanxi 710054; ${ }^{2}$ Department of Orthopedics and Traumatology, The First Affiliated Hospital of Zhejiang Chinese Medicine University, Hangzhou, Zhejiang 310006, P.R. China
\end{abstract}

Received July 26, 2015; Accepted January 28, 2016

DOI: $10.3892 /$ ijmm.2016.2522

\begin{abstract}
In order to investigate the role which sirtuin-6 (SIRT6) plays in lumbar spinal epidural fibrosis (EF) and scar formation in vitro and in vivo, SIRT6 and transforming growth factor $\beta$ (TGF- $\beta$ ) protein levels in the lumbar disc of patients were detected using western blotting in patients who had undergone a laminectomy. The results demonstrated that SIRT6 expression was significantly reduced in the lumbar discs of patients in whom an epidural scar had formed, but the expression pattern of TGF- $\beta 2$ was much higher. Subsequently, a pcDNA-SIRT6 expression vector was constructed and transfected into the primary fibroblasts isolated from the epidural scars. Flow cytometric and MTT analyses indicated that overexpression of SIRT6 suppressed the proliferation of the fibroblasts, and TGF- $\beta 2$ and interleukin- $1 \alpha$ expression, as well as collagen type I ( $\mathrm{Col} \mathrm{I})$ production. The results of bioinformatics and molecular biological analyses demonstarted that TGF- $\beta 2$ was a target of microRNA-21 (miR-21) and SIRT6 overexpression suppressed the levels of TGF- $\beta 2$ through promoting the expression of miR-21. Finally, by injecting the pcDNA-SIRT6 vector, it was possible to observe that SIRT6 suppressed EF and epidural scar formation in vivo. In conclusion, we noted that SIRT6 suppressed EF and epidural scar formation in vitro and in vivo. It was also noted that SIRT6 overexpression suppressed TGF- $\beta 2$ levels by promoting the expression of miR-21.
\end{abstract}

Correspondence to: Dr Xiaochen Fan, Department of Traditional Chinese Medicine Orthopedics Diagnosis and Treatment Center, Honghui Hospital, Xi'an Jiaotong University Health Science Center, 555 Youyi East Road, Xi'an, Shaanxi 710054, P.R. China

E-mail: xiaochenfan163@163.com

*Contributed equally

Key words: sirtuin-6, epidural fibrosis, microRNA-21, transforming growth factor $\beta$

\section{Introduction}

Epidural fibrosis (EF), which causes spinal epidural adhesion and scarring, is frequently found in the epidural space and contributes greatly to postoperative pain and recurrent lumbar disc herniation after a laminectomy $(1,2)$. Various agents or mechanical barriers for preventing EF have been studied both in animal models and humans, including autologous fat grafts, Adcon-L, polytetrafluoroethylene membranes, and fibrinolytic agents (3). However, it is still difficult to treat patients with established spinal EF.

$\mathrm{EF}$ formation is known to be a complicated process, involving hypernomic proliferation of fibroblasts, the increased expression of inflammatory cytokines, the exorbitant production of collagens and other changes in cellular and molecular components $(4,5)$. After laminectomy, fibrous connective tissues adjacent to the laminar traumas are activated to repair the wound; fibroblast proliferation and inflammatory cytokine release are greatly increased during the repair process (6). As a major cell component involved in this repair, fibroblasts are driven to excessively proliferate and produce collagens (mainly $\mathrm{Col}$ I) by the increased growth factors and inflammatory mediators (7).

Transforming growth factor $\beta$ (TGF- $\beta$ ) is a secreted protein that controls proliferation, differentiation, metastasis and other functions in the majority of types of cells (8). TGF- $\beta$ has been shown to be involved in the progression of many chronic inflammation-related diseases, including diabetes, multiple fibrosis/sclerosis and also in scar formation (9-12). Of the members of the TGF- $\beta$ family, TGF- $\beta 1$ and TGF- $\beta 2$ are believed to play a central role in the fibrosis and scar formation in tissues (13). During these processes, these were upregulated, and induced the production of multiple growth factors, inflammatory cytokines and collagens (14). A previous study on TGF- $\beta 1$ and TGF- $\beta 2$ expression in mice with spinal injury revealed that TGF- $\beta 1$ was induced at an early stage while TGF- $\beta 2$ was induced at a later stage (15). Another study demonstrated that expression of TGF- $\beta 2$ but not TGF- $\beta 1$ was correlated with the deposition of scar tissue in the spinal lesion (16). These studies revealed that TGF- $\beta 2$ plays a pivotal role in the recovery of patients with spinal lesions.

Sirtuins (SIRTs) are a highly conserved $\mathrm{NAD}^{+}$-dependent deacetylase family that plays multiple roles in metabolism, cell 
apoptosis, cell fate determination and lifespan regulation (17). As an important member of the SIRT family, SIRT6 has been proven to deacetylate many important proteins involved in physiological and pathological processes, and plays a role in DNA repair, metabolism and aging (18). Depletion of SIRT6 has been shown to result in the suppression of cell apoptosis, cellular senescence, DNA damage, and telomere dysfunction in many types of cells (19-21). However, the role and mechanism of SIRT6 in spinal EF have not been studied in depth.

In the present study, we found that SIRT6 expression was significantly reduced the lumbar disc of the patients in whom an epidural scar formed, displaying an opposite expression pattern to TGF- $\beta 2$. Overexpression of SIRT6 in primary epidural fibroblasts suppressed cell proliferation, TGF- $\beta 2$ and interleukin-1 $\alpha$ (IL-1 $\alpha$ ) expression, and Col I production. Our results of the bioinformatics and molecular biological analyses revealed that SIRT6 overexpression suppressed TGF- $\beta 2$ levels through inducing the expression of microRNA-21 (miR-21). Finally, a study of pcDNA-SIRT6 vector injection indicated that SIRT6 could suppress EF and epidural scar formation in vivo.

\section{Patients and methods}

Patients and sampling. This study enrolled 96 post-laminectomy patients with lumbar disc herniation, of which 48 patients developed an epidural scar and the other 48 patients did not develop an epidural scar. Their lumbar disc tissues were sampled during the laminectomy, and the removed tissues were collected, labeled and saved at $-80^{\circ} \mathrm{C}$ for future use. This study was approved by the Ethics Committee of Xi'an Red Cross Hospital (Xi'an, China). All participants provided written informed consent.

Cell culture and transfection. Samples of epidural scar tissues were taken, and human primary epidural fibroblasts were isolated from these as described previously (22). The primary fibroblasts were obtained from the epidural scars using the enzymatic digestion method in a sterile superclean bench. A total of $5 \mathrm{mg}$ obtained tissues were cut into small pieces (approximately $1 \mathrm{~mm}^{3}$ ) and mixed with $3 \mathrm{ml} 0.2 \%$ collagenase II. The mixture was incubated at $37^{\circ} \mathrm{C}$ in a water bath for $50 \mathrm{~min}$. An equal volume of Dulbecco's modified essential medium (DMEM)/ F12 with fetal bovine serum (FBS; Invitrogen, Grand Island, NY, USA) was added to terminate the digestion. The mixture was then transferred to sterile tubes and centrifuged at $500 \mathrm{xg}$ for $7 \mathrm{~min}$. We resuspended the precipitate with $2 \mathrm{ml}$ medium and repeated the centrifugation and resuspension twice. The isolated cells were incubated in DMEM (Life Technologies, Gaithersburg, MD, USA) containing 10\% FBS, $100 \mu \mathrm{g} / \mathrm{ml}$ streptomycin and penicillin. Fibroblasts from passages 4 to 6 were used in the subsequent experiments. The cells were incubated in a humidified incubator with an atmosphere of $95 \%$ air and $5 \% \mathrm{CO}_{2}$ at $37^{\circ} \mathrm{C}$. On reaching $80 \%$ confluence, $1 \mu \mathrm{g}$ pcDNA-SIRT6 or $1 \mu \mathrm{g}$ pcDNA3.1 empty vector (vector), 6 pmol miR-21 mimic or inhibitor were transfected into the fibroblasts with Lipofectamine ${ }^{\circledR} 3000$ (Invitrogen Life Technologies, Carlsbad, CA, USA) according to the manufacturer's instructions. The medium was changed every 3 days.

The pcDNA-SIRT6 overexpression vector and empty vector were kindly presented by Professor David Lohnes (Department of Cellular and Molecular Medicine, University of Ottawa, Ottawa, ON, Canada). The 2'-OMe modified miR-21 mimic/inhibitor or NC mimic/inhibitor were synthesized and confirmed to be effective by Invitrogen Life Technologies.

Cell proliferation and apoptosis analyses. Cell proliferation was evaluated using an MTT assay (Sigma, St. Louis, MO, USA). The cells were seeded in 12-well culture plates at $5 \times 10^{4} /$ well, and then transfected with the indicated vectors or siRNA. The cells were incubated for $0,24,48$ and 72 before adding the MTT reagent to each well at a final concentration of $0.5 \mathrm{mg} / \mathrm{ml}$ and incubated at $37^{\circ} \mathrm{C}$ for $4 \mathrm{~h}$. After medium removal, $500 \mu \mathrm{l}$ dimethylsulfoxide was added to each well. Viable cells were measured at an absorbance of $550 \mathrm{~nm}$ wavelength using a microplate reader (Bio-Rad Laboratories, Inc., Hercules, CA, USA).

In the present study, apoptosis was detected using annexin V/ propidium iodide (PI) dual staining, which was followed by flow cytometric analysis using a flow cytometer [Cytomics ${ }^{\mathrm{TM}}$ FC500 (Beckman Coulter Ltd., Brea, CA, USA)].

Quantitative polymerase chain reaction ( $q P C R$ ). Total RNA was isolated using TRIzol reagent (Invitrogen Life Technologies) following the manufacturer's instructions. The integrity of RNA was checked by electrophoresis on $1.0 \%$ agarose gel with ethidium bromide staining. qPCR reactions were carried out in a final volume of $25 \mu$ l, using SYBR Premix Ex Taq (Takara Bio, Dalian, China), $0.4 \mathrm{mM}$ of each primer, and $200 \mathrm{ng}$ of cDNA template. Primers used in the reactions are as follows: SIRT6 (F: 5'-GTTAGCCATCAAGACGC-3', R: 5'-TCAGGG ATACAGGGATG-3'); TGF- 32 (F: 5'-ACACTCGCTGCGTAC TCAG-3', R: 5'-AGTCTCTCTTGCTGCTGAC-3'); IL-1 $\alpha$ (F: 5'-CTAGCTATCAGGAACATTTAT-3', R: 5'-TGCTCATGC CTCGTCCT-3'); $\beta$-actin (5'-ACGGGACCTAATGAAA CTC-3', R: 5'-CAAGAAGATGCGGCTGT-3'); pri-, pre- and mature miR-21 stem-loop primer and the quantitative primers and U6 RNA primers were designed and produced by Guangzhou RiboBio Co., Ltd. (Guangzhou, China). Each individual sample was run in triplicate wells. The reactions were initially denatured at $95^{\circ} \mathrm{C}$ for 3 min followed by 35 cycles of $95^{\circ} \mathrm{C}$ for $15 \mathrm{sec}, 60^{\circ} \mathrm{C}$ for $1 \mathrm{~min}$. The change of transcript abundance of all tested genes was calculated using the $2^{-\Delta \Delta \mathrm{Ct}}$ method. mRNA was normalized to $\beta$-actin; miR-21 transcripts were normalized to U6 RNA.

Western blotting. A total of $50 \mu \mathrm{g}$ of protein of each sample were separated by $12 \%$ SDS-PAGE and electro-transferred to a PVDF membrane (Millipore Corp., Billerica, MA, USA) for western blotting. The following primary antibodies (purchased from Abcam, Cambridge, UK) were used: anti-Col I (1:300; ab34710), anti-SIRT6 (1:300; ab88494), anti-TGF- 32 (1:500; ab66045) and anti- $\beta$-actin (1:200; ab189073), which was used as the internal reference. After incubation with the appropriate horseradish peroxidase-conjugated secondary antibody (goat anti-rabbit $\operatorname{IgG}$, 1:3,000, ab7090; Abcam), proteins were detected using chemiluminescence reagent (ECL; Invitrogen Life Technologies) in a ChemiDoc XRS imaging system and analyzed with Quantity One software (both from Bio-Rad Laboratories, Inc.).

ChIP analysis. The regulatory association between SIRT6 and miR-21 was calculated using the online platform ChIPBase (http:// deepbase.sysu.edu.cn/chipbase/), which is an integrated resource 
for decoding transcription factor binding maps, expression profiles and the transcriptional regulation of non-coding RNAs and protein-coding genes from ChIP-seq data. The targeting association between miR-21 and TGF- $\beta 2$ was predicted with TargetScan (http://www.targetscan.org/), an online resource.

3'-UTR luciferase reporter assay. The 3'-UTR of TGF- $\beta 2$ mRNA was amplified by PCR, using primers linked with the XhoI and NotI restriction sites, respectively. Both the primers were designed and synthesized by Genscript Co., Ltd., (Nanjing, China). The PCR products were excised with NotI and XhoI and inserted into the pGL-2 vector (Promega, Madison, WI, USA) at the 3'-end of the Renilla gene CDS. Firefly luciferase activity was used as the internal control. The 3'-UTR dual-luciferase vectors were transfected or co-transfected with the miR-21 mimic or pcDNA-TGF- $\beta 2$ expression vector into the primary fibroblasts (100\% confluence) using X-tremeGENE 9 DNA transfection reagent (Roche, Basel, Switzerland). The medium was changed $6 \mathrm{~h}$ later. Cells were incubated for another $48 \mathrm{~h}$ with commercial cell lysis buffer (Merck, Whitehouse Station, NJ, USA). The luciferase activity was measured using a luminometer (Promega) according to the manufacturer's instructions.

Lumbar disc herniation models and injection. In the present study, a Xenopus tropicalis model of lumbar disc herniation was established using the method of caudal vertebral pulposus transplantation, as described previously (23). The animals (aged approximately 12 weeks; purchased from the Laboratory Animal Center, Beijing Institute of Genetics and Development, Chinese Academy of Sciences, Beijing, China) underwent a laminectomy and subsequently those which developed epidural fibrotic scars were selected and used in the following experiment. The Xenopus tropicalis were sacrificed using a dissecting needle inserted into the vertebral foramen. For caudal vertebral pulposus transplantation, the animals were anaesthesized with $40 \mathrm{mg} / \mathrm{kg}$ after adaptive feeding for 1 week. We cut the tail vertebrae at the root of the tail (about $1 \mathrm{~cm}$ from the anus), and the incision was then sutured. The caudal nucleus pulposus was removed, and we weighed $10 \mathrm{mg}$ of the pulposus with an analytical balance (Pingxuan Scientific Instrument Co., Ltd., Shanghai, China). We then cut open the back of the Xenopus tropicalis, removed the spinous processes and lamina of L4-L6, and gently set the obtained pulposus on the nerve root. Finally, the incisions were sutured. The miR-21 inhibitor $(1 \mu \mathrm{g} / \mathrm{g}$ body weight) or pcDNA-SIRT6 (5 $\mu \mathrm{g} / \mathrm{g}$ body weight) was locally injected into the animals around the surgical site. Over a 6-week period, the injection was administered once a day. Subsequently, they were sacrificed, and their epidural scar tissues were removed carefully, and the mass of total scars and Col I tissues was examined using an electronic analytical balance (Pingxuan Scientific Instrument Co., Ltd.), and staining analyses were then undertaken.

Masson's and immunohistochemical staining. Masson's trichrome staining was used to analyze the collagen fibers in the epidural scars in this study. The tissues were deparaffinized and rehydrated using 100\% alcohol, 95\% alcohol, 70\% alcohol and then washed in distilled water. For formalin-fixed tissue, these were re-fixed in Bouin's solution for $1 \mathrm{~h}$ at $56^{\circ} \mathrm{C}$ to improve staining quality although this step is not absolutely neces- sary. They were rinsed under running tap water for 5-10 min to remove the yellow color and then stained using Weigert's iron hematoxylin working solution for $10 \mathrm{~min}$. The tissues were rinsed in running warm tap water for $10 \mathrm{~min}$, washed in distilled water and stained in Biebrich scarlet-acid fuchsin solution for 10-15 min. They can thus be saved for future use, and our samples were washed in distilled water. They were differentiated in phosphomolybdic-phosphotungstic acid solution for 10-15 min or until the collagen was not red. The sections were transferred directly (without rinsing) to aniline blue solution and stained for 5-10 min. They were rinsed briefly in distilled water and differentiated in $1 \%$ acetic acid solution for 2-5 min and subsequently washed in distilled water. These were dehydrated rapidly using $95 \%$ ethyl alcohol, absolute ethyl alcohol (this step removes Biebrich scarlet-acid fuchsin staining) and cleared in xylene. They were mounted with resinous mounting medium. Finally, the stained samples were observed under a microscope (Senben Electronic Technology Co., Ltd., Shenzhen, China).

Vimentin immunohistochemical staining was used to evaluate the degree of inflammation in the epidural scars. Vimentin in the cells was stained using StreptAvidin-Biotin Complex-myoglobin (SABC-Mb), and the images were captured using a CQ-L323 phase contrast microscope (Senben Electronic Technology Co., Ltd.).

Statistical analysis. All data were obtained from $\geq 3$ independent experiments. Values are expressed as the means \pm standard error of the means. Statistics were calculated using SPSS 18.0 (SPSS, Inc., Chicago, IL, USA). Multiple comparisons were assessed by one-way ANOVA followed by Dunnett's tests. A P-value $<0.05$ was considered to indicate a statistically significant difference.

\section{Results}

Expression of SIRT6 is reduced in the lumbar disc tissues of the patients with an epidural scar. As mentioned in Materials and methods, following laminectomy, the removed lumbar disc tissues were collected, labeled and saved at $-80^{\circ} \mathrm{C}$. Two years later, an investigation was undertaken to confirm whether each patient had an epidural scar and whether there had been a relapse of lumbar disc herniation. A total of 48 scar-free patients and 48 patients who developed an epidural scar were randomly selected. Expression of SIRT6 and TGF- $\beta 2$ in their lumbar disc tissues sampled previously was detected using western blotting, and the results showed that SIRT6 levels in the patients with epidural scarring were significantly lower than those of scar-free patients (Fig. 1A). By contrast, TGF- $\beta 2$ expression in the patients who suffered an epidural scar was much higher than that of scar-free patients (Fig. 1B). These results suggested that lumbar disc SIRT6 expression is associated with epidural scar formation and also TGF- $\beta 2$ expression.

SIRT6 suppresses fibroblast proliferation, TGF- $\beta 2$ and IL-1 $\alpha$ expression, and Col I production. In order to explore the role of SIRT6 in EF and scar formation in vitro, epidural scar samples were taken from the patients, and primary fibroblasts were isolated using the collagenase I digestion method. A pcDNA-SIRT6 expression vector was built and transfected 
A

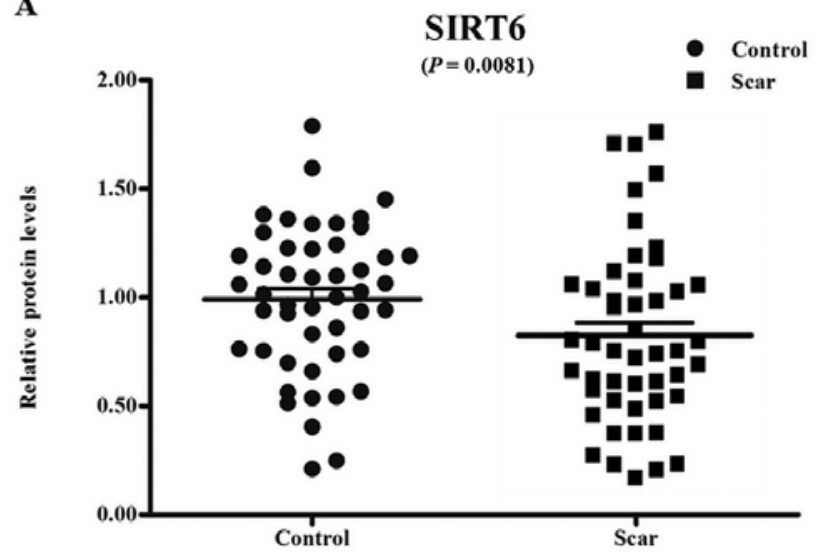

B

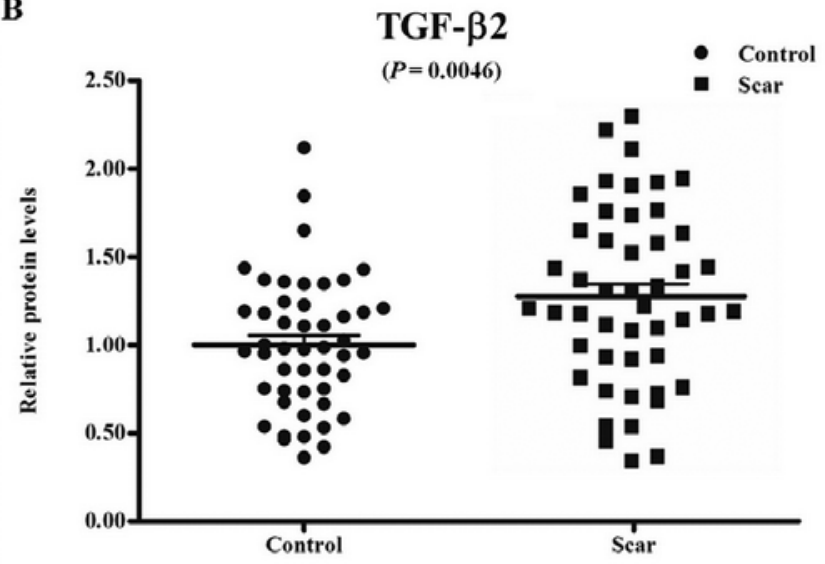

Figure 1. Expression of sirtuin-6 (SIRT6) was lower in patients who developed epidural scars after laminectomy. (A) Comparison of SIRT6 protein levels between patients who developed epidural scars and those who did not. (B) Comparison of transforming growth factor $\beta$ (TGF- $\beta$ ) protein levels between patients who developed epidural scars and those who did not. Following laminectomy, 48 scar-free patients and 48 patients who developed an epidural scar were randomly selected. Expression of SIRT6 and TGF- $\beta 2$ in the lumbar disc tissues samples was detected using western blotting. Control, non-scarred patients.

A

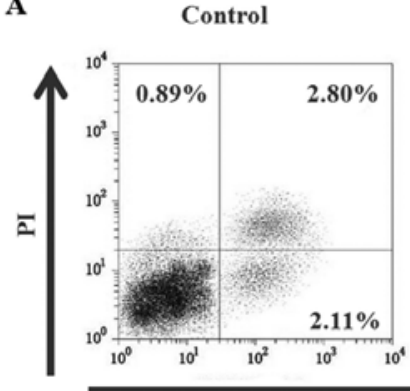

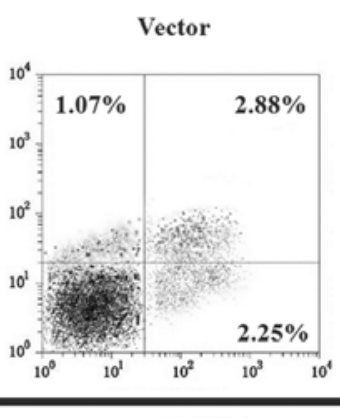

Annexin V-FITC
B



C

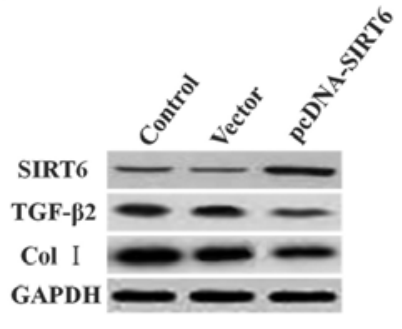

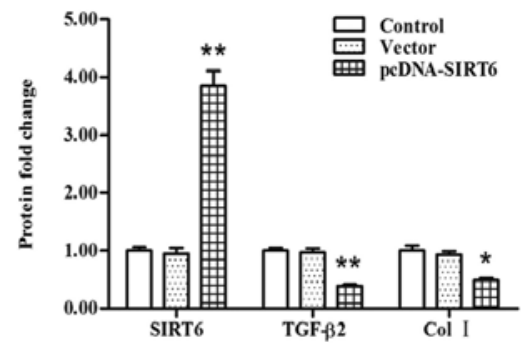

D

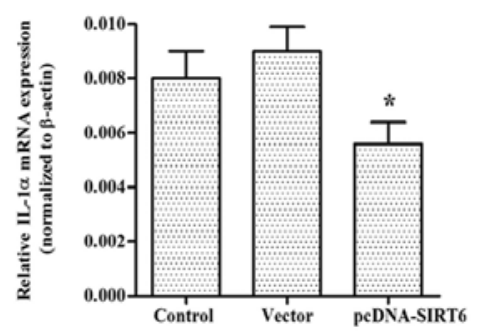

Figure 2. Overexpression of sirtuin-6 (SIRT6) suppressed epidural fibrosis in vitro. (A) Cell apoptosis detected with propidium iodide/annexin V dual staining after pcDNA-SIRT6 transfection for $72 \mathrm{~h}$. (B) Cell viability detected with MTT method after pcDNA-SIRT6 transfection for $72 \mathrm{~h}$. (C) Expression of SIRT6, transforming growth factor $\beta$ (TGF- $\beta$ ) and collagen type I (Col I) proteins detected with western blotting after pcDNA-SIRT6 transfection for $72 \mathrm{~h}$. (D) Expression of interleukin (IL)- $1 \alpha$ mRNA detected via qPCR. ${ }^{*} \mathrm{P}<0.05,{ }^{* *} \mathrm{P}<0.01$ vs. the control. Control, untreated fibroblasts.

into the primary cells. After incubation for $72 \mathrm{~h}$, the cells were stained with PI/annexin V followed by flow cytometric analysis. The results showed that the proportion of viable cells was reduced by pcDNA-SIRT6 transfection but there were no marked changes in the proportion of early apoptotic cells among the groups (Fig. 2A). MTT analysis also demonstrated that pcDNA-SIRT6 transfection reduced the viability of the cells (Fig. 2B). The levels of TGF- $\beta 2$ and Col I proteins, as well as IL-1 $\alpha$ mRNA, were determined by western blotting and qPCR, respectively. The data indicated that SIRT6 overexpression caused a significant decrease in TGF- $\beta 2$ and $\mathrm{Col}$ I protein levels (Fig. 2C) and the IL-1 $\alpha$ mRNA level (Fig. 2D). These data indicated that SIRT6 suppressed EF, which manifested as suppression in fibroblast proliferation, TGF- $\beta 2$ and IL- $1 \alpha$ expression, as well as Col I production.

TGF- $\beta 2$ is a target of miR-21, and SIRT6 promotes the expression of miR-21 in primary fibroblasts. Subsequently, the mechanism of SIRT6 suppression of EF was investigated. Bioinformatics analysis using TargetScan revealed that there was a complete match between the miR-21 seed sequence and the 3'UTR of TGF- $\beta 2$ mRNA, and the match was highly conserved among species (Fig. 3A and B). In order to validate the target relationship, the full length of the $3^{\prime} \mathrm{UTR}$ of TGF- $\beta 2$ 
$\mathbf{A}$

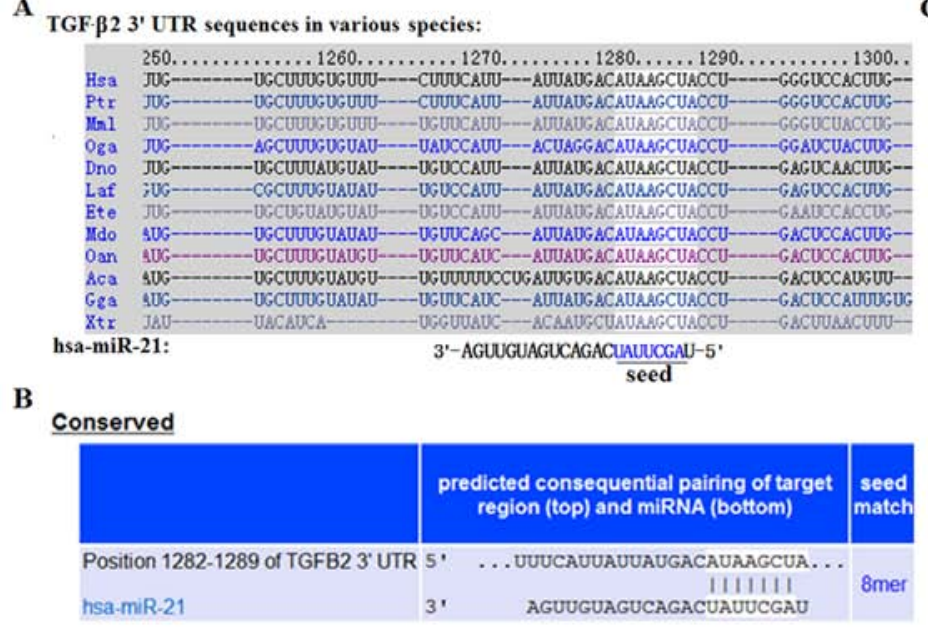

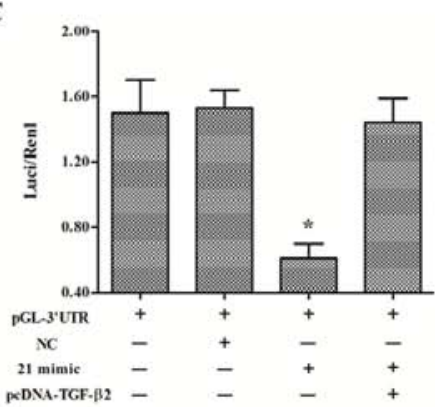



D
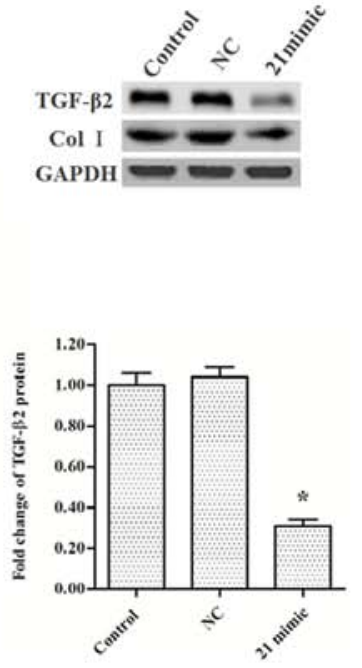

Figure 3. Bioinformatics and luciferase reporter gene assays for targeting the relationship of microRNA-21 (miR-21) and transforming growth factor $\beta$ (TGF- $\beta$ ). (A) Conservation of 3'UTR sequences of TGF- $\beta$ mRNA among species. (B) The output of TargetScan concerning the match of miR-21 seed sequence and the 3'UTR of TGF- $\beta 2$ mRNA (underlined). (C) Luciferase reporter gene assay for targeting the relationship of miR-21 and TGF- $\beta 2$. (D) TGF- $\beta 2$ and collagen type I (Col I) protein expression was reduced after miR-21 mimic transfection for $72 \mathrm{~h}$. " $\mathrm{P}<0.05$ vs. the control. Control, untransfected cells; NC, cells transfected with oligonucleotide that was the same size as miR-21 mimic/inhibitor, but does not target any of the mRNA transcripts.

mRNA was inserted into a pGL3 vector and a luciferase reporter gene assay was performed. The results showed that the miR-21 mimic significantly decreased the fluorescence intensity, while the decrease was rescued by pcDNA-TGF- $\beta 2$ transfection (Fig. 3C). In primary fibroblasts, miR-21 mimic transfection caused a marked reduction in TGF- $\beta 2$ and Col I protein expression (Fig. 3D). Subsequently, the relationship between miR-21 and SIRT6 was predicted using ChIPBase. The results revealed that SIRT6 was likely to be a transcription factor of miR-21 (Fig. 4A). In addition, the overexpression of SIRT6 increased the expression of pri-, pre- and mature miR-21 transcripts (Fig. 4B). These data demonstrated that SIRT6 negatively regulated TGF- $\beta 2$ expression and fibrosis by promoting miR-21 expression.

SIRT6 suppresses EF and scar formation after laminectomy in the Xenopus tropicalis model of lumbar disc herniation. The role of SIRT6 in the suppression of EF and scar formation was investigated in vivo. A Xenopus tropicalis model of lumbar disc herniation was established with caudal vertebral pulposus transplantation. The pcDNA-SIRT6 vector or miR-21 inhibitor were locally injected into the models around the laminectomy site every day. After 6 weeks, the Xenopus models were sacrificed and their epidural scar tissues were removed carefully. The mass of the scars was examined using an electronic analytical balance. The results showed that pcDNA-SIRT6 injection significantly reduced the mass of the total scar and also Col I (Fig. 5A and B). The rate of Col I/total scar was also reduced by pcDNA-SIRT6 injection (Fig. 5C). By contrast, injection with the miR-21 inhibitor increased the total mass of the scar, Col I and the proportion of the Col I/total scar (Fig. 5A-C). Masson's staining analysis showed that the fibers in the pcDNA-SIRT6-injected group were more scattered and slightly stained (Fig. 6A), suggesting a lesser degree of fibrosis. Moreover, the number of vimentin positive $\left(\right.$ vimentin $\left.^{+}\right)$ cells in the pcDNA-SIRT6 injection group was much smaller than the control (Fig. 6B). By contrast, injection of the miR-21 inhibitor exerted the opposite effect to pcDNA-SIRT6 injection (Fig. 6A and B). These data indicate that SIRT6 had a suppressive effect on EF and scar formation in vivo.

\section{Discussion}

As an important member of the TGF- $\beta$ family, TGF- $\beta 1$ has been noted to be the master transcription factor of tissue fibrosis (24). The role of TGF- $\beta 2$ in fibrosis has caught the attention of researchers: previous studies have indicated that TGF- $\beta 2$ acted synergistically with TGF- $\beta 1$ in the fibrotic pathway in certain types of cells and even regulated fibrosis-related biological processes, including spinal fibrosis $(16,25,26)$. In the present study, we found that TGF- $\beta 2$ was markedly upregulated in patients who developed epidural scars, and we noted a novel SIRT6/miR-21 pathway and the inhibition of EF and scar formation upon SIRT6 vector transfection.

The suppression of cell apoptosis and cellular senescence means that SIRT6 acts as a critical regulator in growth inhibition in many types of normal and cancerous cells; it has previously been noted that SIRT6 plays a protective role in the progression of many chronic illnesses including obesity, tumorigenesis, fatty liver, diabetes and cardiac hypertrophy $(27,28)$. Previous studies have revealed that SIRT6 also plays a suppressive role in liver and cardiac inflammation or fibrosis $(27,29)$. However, the effect of SIRT6 on spinal EF had not received so much attention. In the present study, we noted a decreased expression of SIRT6 in patients who developed epidural scars after laminectomy. Our in vitro SIRT6 overexpression study indicated that SIRT6 suppressed epidural fibrotic scar formation, as was clear from our examination of fibroblast proliferation, TGF- $\beta 2$ 


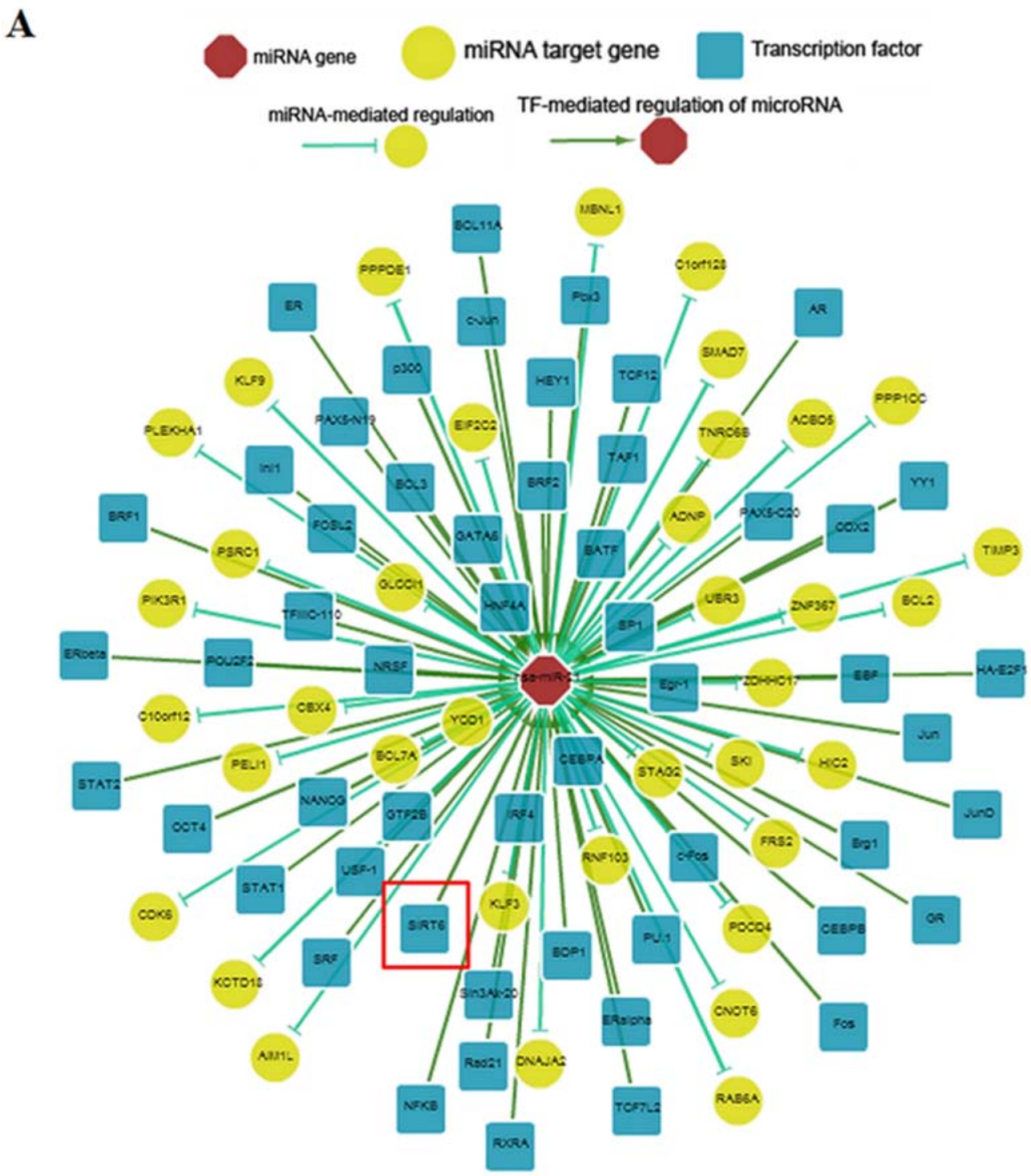

B

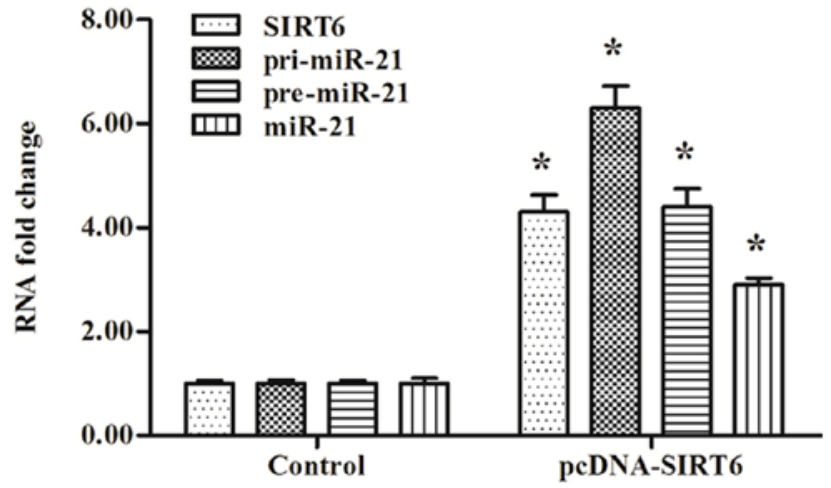

and IL-1 $\alpha$ expression, and Col I production. To the best of our knowledge, this is the first report on the role of SIRT6 and its regulation of spinal $\mathrm{EF}$ and scar formation.

The action of SIRT6 in terms of regulating expression or activity of other genes is largely dependent on its deacetylase activity. For instance, SIRT6 overexpression has been shown to
Figure 4. Sirtuin-6 (SIRT6) was a promoter of microRNA-21 (miR-21) in human primary epidural fibroblasts. (A) The transcription factor (TF)-miRNAs network integrated by online platform ChIPBase. (B) Overexpression of SIRT6 promoted expression of pri-, pre- and mature miR-21 transcripts. Human primary epidural fibroblasts were isolated and seeded in 6-well plates. On reaching $80 \%$ confluence, $1 \mu \mathrm{g}$ pcDNA-SIRT6 was transfected into the fibroblasts with Lipofectamine $^{\circledR} 3000$. After transfection for $72 \mathrm{~h}$, expression of miR-21 transcripts was detected using qPCR. ${ }^{\mathrm{P}}<0.05$ vs. the control (untransfected cells). induce apoptosis through p53 and p73 activation in many cancer cells (19). However, deacetylating proteins at a post-translational level was not the only functional mechanism of SIRT6. A recent study showed that SIRT6 promoted FoxO1 nuclear exclusion and had a significant effect on gluconeogenesis (30). In the present study, our ChIPBase analysis and detection of 
A

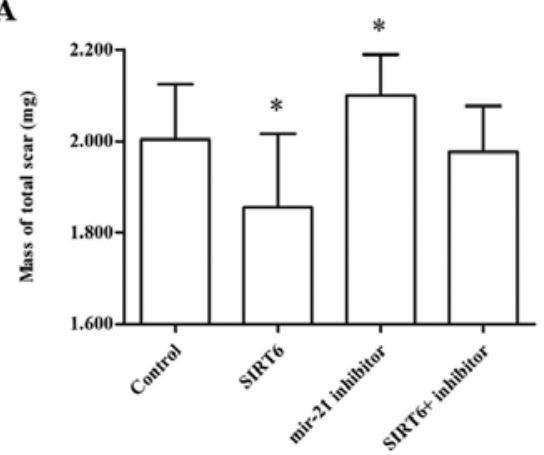

B

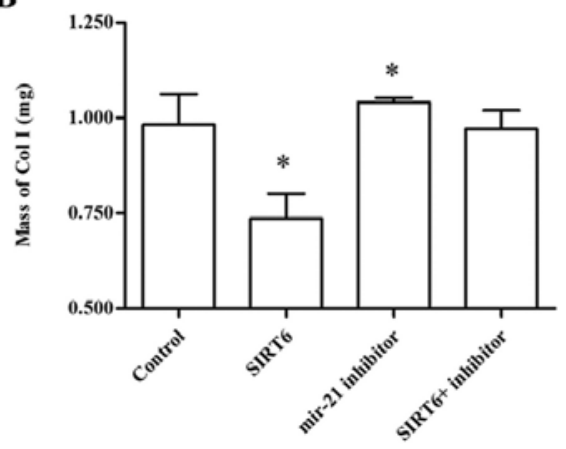

C



Figure 5. pcDNA-sirtuin-6 (SIRT6) vector injection minimizes epidural scar and collagen type I (Col I) proportion in vivo. (A) Total mass of epidural scar was minimized by pcDNA-SIRT6 injection. (B) Total mass of Col I in the epidural scar was reduced by pcDNA-SIRT6 injection. (C) Col I proportion in the scarred tissue was reduced by the pcDNA-SIRT6 injection. The pcDNA-SIRT6 $(5 \mu \mathrm{g} / \mathrm{g}$ body weight), or microRNA-21 (miR-21) inhibitor (1 $\mu \mathrm{g} / \mathrm{g}$ body weight) was locally injected into the Xenopus tropicalis model of lumbar disc herniation around the laminectomy site once a day. After 6 weeks, the mass of total scars and Col I tissues was examined using an electronic analytical balance. ${ }^{*} \mathrm{P}<0.05$ vs. the control (untransfected cells).

A



B

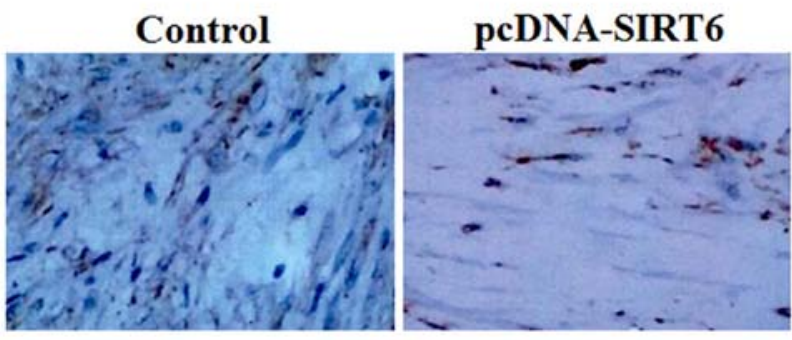

pcDNA-SIRT6
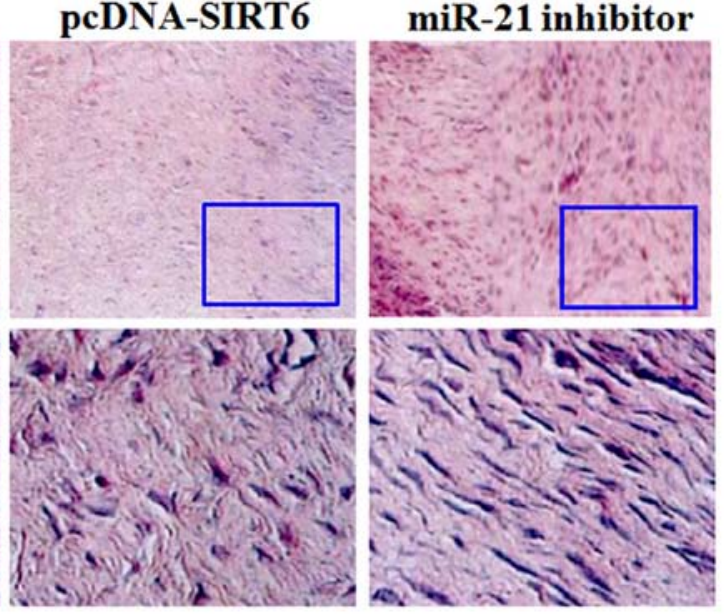

miR-21 inhibitor

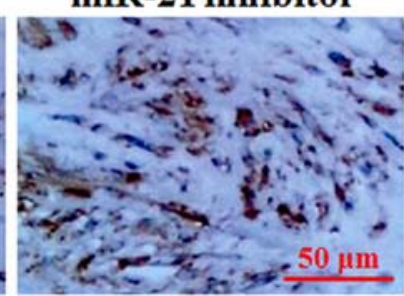

Figure 6. Fibrosis and inflammation are reduced by pcDNA-sirtuin-6 (SIRT6) vector injection in vivo. (A) Masson's staining for collagen fibers (blue). (B) Immunohistochemical staining for vimentin in the epidural scar. The pcDNA-SIRT6 vector or miR-21 inhibitor was locally injected into the Xenopus models around the laminectomy site every day. After 6 weeks, the Xenopus models were sacrificed and their epidural scar tissues were removed carefully. Masson's staining and immunohistochemical staining for vimentin were used to evaluate the degree of fibrosis and inflammation, respectively. Control, untransfected cells

miR-21 transcripts after SIRT6 overexpression showed that SIRT6 is likely a transcription factor for miR-21. We suggest that this is a novel mechanism for SIRT6 regulation of other genes. Moreover, the targeting relationship between miR-21 and TGF- $\beta 2$ is also a novel finding of this study.

Animal models of lumbar disc herniation are an important tool for the exploration of treatment methods and prognosis evaluation. The animal model used in this study was established using the method of caudal nucleus pulposus transplantation (31), which is generally accepted in research on lumbar disc herniation. Although it would have been optimal to establish the model using animals which are closer to humans, considering both the animal experimental cost and the conservation of the TGF- $\beta 2$ gene, we finally determined to use Xenopus tropicalis. This is more convenient for obtaining epidural fibrotic scar tissue.

In conclusion, we examined the role and mechanisms of SIRT6 in suppressing postoperative epidural scar formation. We showed that SIRT6 promoted the expression of miR-21 and then suppressed TGF- $\beta 2$ expression in a targeted manner. 


\section{References}

1. Alkalay RN, Kim DH, Urry DW, Xu J, Parker TM and Glazer PA: Prevention of postlaminectomy epidural fibrosis using bioelastic materials. Spine 28: 1659-1665, 2003.

2. Li C, Wang H, Liu H, Yin J, Cui L and Chen Z: The prevention effect of poly (L-glutamic acid)/chitosan on spinal epidural fibrosis and peridural adhesion in the post-laminectomy rabbit model. Eur Spine J 23: 2423-2431, 2014.

3. Zhang C, Kong X, Ning G, Liang Z, Qu T, Chen F, Cao D, Wang T, Sharma HS and Feng S: All-trans retinoic acid prevents epidural fibrosis through NF- $\mathrm{KB}$ signaling pathway in post-laminectomy rats. Neuropharmacology 79: 275-281, 2014.

4. Manoj Sharma, Rashmi Dahima and Anil Kumar Gupta: A review on role of peridural fibrosis and its diagnosis. Journal of Biomedical and Pharmaceutical Research 2, 81-87, 2013.

5. Spiegelberg L, Swagemakers SM, Van Ijcken WF, Oole E, Wolvius EB, Essers J and Braks JA: Gene expression analysis reveals inhibition of radiation-induced TGF $\beta$-signaling by hyperbaric oxygen therapy in mouse salivary glands. Mol Med 20: 257-269, 2014.

6. Sun Y, Yan LQ, Liang Y, Li XL, Cao XJ and Lu C: Reduction of epidural scar adhesion by topical application of simvastatin after laminectomy in rats. Eur Rev Med Pharmacol Sci 19: 3-8, 2015.

7. Wang Z, Wang Y, Xie P, Liu W and Zhang S: Calcium channel blockers in reduction of epidural fibrosis and dural adhesions in laminectomy rats. Eur J Orthop Surg Traumatol 24 (Suppl 1): S293-S298, 2014.

8. Chang H, Brown CW and Matzuk MM: Genetic analysis of the mammalian transforming growth factor- $\beta$ superfamily. Endocr Rev 23: 787-823, 2013.

9. Yan J, Zhang H, Yin Y, Li J, Tang Y, Purkayastha S, Li L and Cai D: Obesity- and aging-induced excess of central transforming growth factor- $\beta$ potentiates diabetic development via an RNA stress response. Nat Med 20: 1001-1008, 2014.

10. Avouac J, Palumbo K, Tomcik M, Zerr P, Dees C, Horn A, Maurer B, Akhmetshina A, Beyer C, Sadowski A, et al: Inhibition of activator protein 1 signaling abrogates transforming growth factor $\beta$-mediated activation of fibroblasts and prevents experimental fibrosis. Arthritis Rheum 64: 1642-1652, 2012.

11. Bhattacharyya S, Kelley K, Melichian DS, Tamaki Z, Fang F, Su Y, Feng G, Pope RM, Budinger GR, Mutlu GM, et al: Toll-like receptor 4 signaling augments transforming growth factor- $\beta$ responses: a novel mechanism for maintaining and amplifying fibrosis in scleroderma. Am J Pathol 182: 192-205, 2013.

12. Heldin $\mathrm{CH}$ and Moustakas A: Role of Smads in TGF $\beta$ signaling. Cell Tissue Res 347: 21-36, 2012.

13. Huang $C$ and Ogawa R: Fibroproliferative disorders and their mechanobiology. Connect Tissue Res 53: 187-196, 2012.

14. Zgheib C, Xu J and Liechty KW: Targeting inflammatory cytokines and extracellular matrix composition to promote wound regeneration. Adv Wound Care (New Rochelle) 3: 344-355, 2014

15. Joko M, Osuka K, Usuda N, Atsuzawa K, Aoyama M and Takayasu M: Different modifications of phosphorylated Smad3C and Smad3L through TGF- $\beta$ after spinal cord injury in mice. Neurosci Lett 549: 168-172, 2013.

16. Lagord C, Berry M and Logan A: Expression of TGF $\beta 2$ but not TGF $\beta 1$ correlates with the deposition of scar tissue in the lesioned spinal cord. Mol Cell Neurosci 20: 69-92, 2002.
17. Lin H: Sirtuins and novel protein post translational modifications. The FASEB Journal 29: 496.1, 2015.

18. Lombard DB, Schwer B, Alt FW and Mostoslavsky R: SIRT6 in DNA repair, metabolism and ageing. J Intern Med 263: 128-141, 2008.

19. Van Meter M, Mao Z, Gorbunova V and Seluanov A: SIRT6 overexpression induces massive apoptosis in cancer cells but not in normal cells. Cell Cycle 10: 3153-3158, 2011.

20. Schwer B, Schumacher B, Lombard DB, Xiao C, Kurtev MV, Gao J, Schneider JI, Chai H, Bronson RT, Tsai LH, et al: Neural sirtuin 6 (Sirt6) ablation attenuates somatic growth and causes obesity. Proc Natl Acad Sci USA 107: 21790-21794, 2010.

21. Etchegaray JP, Zhong L and Mostoslavsky R: The histone deacetylase SIRT6: at the crossroads between epigenetics, metabolism and disease. Curr Top Med Chem 13: 2991-3000, 2013.

22. Shi K, Wang D, Cao X and Ge Y: Endoplasmic reticulum stress signaling is involved in mitomycin C (MMC)-induced apoptosis in human fibroblasts via PERK pathway. PLoS One 8: e59330, 2013.

23. Sekiguchi M, Konno S and Kikuchi S: The effects of a 5-HT2A receptor antagonist on blood flow in lumbar disc herniation: application of nucleus pulposus in a canine model. Eur Spine J 17: 307-313, 2008.

24. Verrecchia F and Mauviel A: Transforming growth factor-beta and fibrosis. World J Gastroenterol 13: 3056-3062, 2007.

25. Kamath VV, Krishnamurthy S, Satelur KP and Rajkumar K: Transforming growth factor- $\beta 1$ and TGF- $\beta 2$ act synergistically in the fibrotic pathway in oral submucous fibrosis: an immunohistochemical observation. Indian J Med Paediatr Oncol 36: 111-116, 2015.

26. Feng Z, Li R, Shi H, Bi W, Hou W and Zhang X: Combined silencing of TGF- $\beta 2$ and Snail genes inhibit epithelial-mesenchymal transition of retinal pigment epithelial cells under hypoxia. Graefes Arch Clin Exp Ophthalmol 253: 875-884, 2015.

27. Xiao C, Wang R-H, Lahusen TJ, Park O, Bertola A, Maruyama T, Reynolds D, Chen Q, Xu X, Young HA, et al: Progression of chronic liver inflammation and fibrosis driven by activation of c-JUN signaling in Sirt6 mutant mice. J Biol Chem 287: 41903-41913, 2012.

28. Swierczynski S, Klieser E, Illig R, Alinger-Scharinger B, Kiesslich T and Neureiter D: Histone deacetylation meets miRNA: epigenetics and post-transcriptional regulation in cancer and chronic diseases. Expert Opin Biol Ther 15: 651-664, 2015.

29. Pillai VB, Sundaresan NR and Gupta MP: Regulation of Akt signaling by sirtuins: its implication in cardiac hypertrophy and aging. Circ Res 114: 368-378, 2014.

30. Zhang P, Tu B, Wang H, Cao Z, Tang M, Zhang C, Gu B, Li Z, Wang L, Yang Y, et al: Tumor suppressor p53 cooperates with SIRT6 to regulate gluconeogenesis by promoting FoxO1 nuclear exclusion. Proc Natl Acad Sci USA 111: 10684-10689, 2014.

31. Iwashina T, Mochida J, Sakai D, Yamamoto Y, Miyazaki T, Ando K and Hotta T: Feasibility of using a human nucleus pulposus cell line as a cell source in cell transplantation therapy for intervertebral disc degeneration. Spine 31: 1177-1186, 2006. 